\title{
APROXIMACIÓN PARA EL ANÁLISIS DE DIBUJOS REALIZADOS POR NIÑOS ENTRE 6 Y 9 AÑOS DE EDAD CON EL. FIN DE CONOCER RASGOS DE SU PERSONALIDAD Y SU PERCEPCIÓN ANTE LA CONSULTA ODONTOLÓGICA
}

\author{
${ }^{1}$ Emma Solangel Reyes C., ${ }^{1}$ Claudia Milena Riveros A., ${ }^{2}$ Lucy Forero O., \\ ${ }^{2}$ Liliana M. Osorio A., ${ }^{3}$ Mercelena Sánchez $M$. \\ ${ }^{1}$ Odontóloga, U. El Bosque, residente II año, Especialización en Odontopediatría, U. Santo Tomás, ${ }^{2}$ Odontóloga, U. Santo Tomás, \\ residente II año, Especialización en Odontopediatría, U. Santo Tomás, ${ }^{3}$ Odontóloga, U. Autónoma de Manizales, residente II año, \\ Especialización en Odontopediatría, U. Santo Tomás.
}

Autor responsable de correspondencia: Emma Solangel Reyes $C$.

Dirección de correo electrónico: emmasolr@hotmail.com

Presentación realizada en el III Simposio de Actualización en Odontología Pediátrica en Bogotá, Noviembre de 2006

\begin{abstract}
RESUMEN
El Odontopediatra es el Especialista con mayor conocimiento en el manejo de la conducta. En su formación es esencial el estudio del comportamiento del niño y cómo éste se puede ver reflejado en las diferentes actividades que realizan. Los dibujos infantiles se consideran una forma de expresión, a veces inconsciente, de sí mismos tal como son o tal como les gustaría ser. Estos representan un lenguaje simbólico que es necesario comprender e interpretar, son la proyección de un mundo significativo al que se le debe dar importancia pues pueden ser un puente de comunicación y de conocimiento. El propósito de este trabajo fue analizar los rasgos generales de la personalidad y la percepción del paciente pediátrico hacia el odontopediatra, a través de dibujos. [Reyes ES, Riveros CM, Forero L, Osorio LM, Sanchez M. Aproximación para el análisis de dibujos realizados por niños entre 6 y 9 años de edad con el fin de conocer rasgos de su personalidad y su percepción ante la consulta odontológica. Ustasalud Odontología 2006; 5: 123 - 131]
\end{abstract}

Palabras clave: Dibujo infantil, Consulta odontológica, Manejo del comportamiento.

ANALYSIS APROACH OF DRAWINGS MADE BY CHILDREN BETWEEN 6 AND 9 YEARS OLD WITH THE PURPOSE OF KNOWING CHARACTERISTICS FROM ITS PERSONAIITY AND ITS PERCEPTION AT THE DENTAL OFFICE

\begin{abstract}
The Pediatric Dentist is the one with a great knowledge in the handling of the children behavior. This behaviour can be seen reflected in the different activities. Children's drawings are consider a way of expression, sometimes unconscious, of themselves as they are or as they would like to be. These represent a symbolic language that is necessary to understand and to interpret, are the projection of a significant world and a communication bridge. The purpose of this work was to analyze the general characteristics of the personality and the perception of the children patient at the pediatric dentist, through drawings.
\end{abstract}

Key words: Children's drawing, Dental office, Behavior management.

Recibido para publicación: 2 de junio de 2006. Aceptado para publicación: 10 de noviembre de 2006.

\section{INTRODUCCIÓN}

El manejo de la conducta es uno de los tópicos que el odontopediatra debe conocer perfectamente ya que el comportamiento del paciente pediátrico influye, notablemente, en la calidad del tratamiento odontológico y en el desarrollo de la consulta como tal. ${ }^{1-4}$

Por lo tanto, es indispensable establecer un vínculo con el niño y su familia para que conozcan y acepten todas las acciones tera- péuticas necesarias. De esta manera, el profesional pone en juego su personalidad, el nivel de tolerancia, la preparación académica y el manejo de sus emociones. ${ }^{1}$

Se han destacado varias maneras para establecer dicho contacto. En los últimos años, ha cobrado gran importancia el dibujo infantil como un elemento de ayuda para conocer la percepción del niño sobre si mismo y sobre su ambiente, y así manejar su comportamiento., 
Los dibujos infantiles empiezan a ser manejados, por los niños, antes del desarrollo del lenguaje y de la escritura. Se consideran una de las actividades más importantes para su desarrollo intelectual; en ellos, expresan su estado psicológico y su bienestar emocional, además son un medio de socialización ya que les permite establecer una relación entre su mundo interno y el exterior. ${ }^{5.6}$

Tanto el tamaño como la disposición espacial de los objetos en el dibujo pueden ser afectados por los sentimientos del menor.? También, se ha descrito que los colores son una forma de expresar sus emociones. ${ }^{8,9}$

Los dibujos pueden ser usados para evaluar el desarrollo, evaluar el conocimiento, diagnosticar abusos o problemas emocionales, intervenciones terapéuticas o, simplemente, como una actividad de juego., ${ }^{5,11}$ Requiere habilidades que se van perfeccionando con la edad y con la práctica; estas habilidades integran la motricidad fina, la coordinación entre ojos y manos, la adquisición del lenguaje y el desarrollo cognitivo. ${ }^{5}$

Lowenfeld y Brittain, en 1970, describieron cuatro etapas en relación con el desarrollo picto-gráfico del niño:

- Etapa de garabateo (2 a 4 años): el niño comienza a dibujar trazos desordenados que se van organizando y controlando a través del tiempo; llegan a ser reconocidos a los 4 años.

- Etapa pre-esquemática (4 a 7 años): crea formas de manera conciente. Se dibujan figuras y objetos de su entorno ubicados sin orden. El color adquiere importancia. ${ }^{9}$

Etapa esquemática (7 a 9 años): hay un concepto definido de la forma, describe parte de su ambiente, se incluye el factor tiempo y pueden aparecer diferentes secuencias de tiempo 0 acciones. El niño usa el color para representar objetos específicos. ${ }^{9}$

- Etapa del realismo (9 a 12 años): los niños empiezan a sentirse miembros de una sociedad y del ambiente en que viven. Sus dibujos son más detallados y proporcionados, tienen perspectivas y movimientos flexibilizando las figuras, diferencias claramente los colores. ${ }^{9,12,13}$

La técnica de análisis de dibujo se debe a muchos métodos ya establecidos sobre el análisis de la personalidad y la teoría psicoanalítica. Su validez se soporta en comprobaciones clínicas en las cuales se hace énfasis en cierto rasgo grafico. ${ }^{6,14}$ Entre los aspectos pertinentes de la propia presentación del sujeto se incluyen el tamaño de la figura, el lugar donde se haya colocado en la hoja, la rapidez del movimiento gráfico, la presión, la solidez y la variabilidad de la línea, la sucesión de las partes dibujadas, el porte, el uso del fondo o los efectos de base, la extensión de los brazos hacia el cuerpo o hacia fuera del mismo, la espontaneidad o la rigidez, si la figura se dibuja de perfil o de frente. ${ }^{5 \cdot 9}$
En el análisis, también, se otorga consideración a las proporciones de cada parte del cuerpo, la tendencia a dejar ciertos datos incompletos, la cantidad de los detalles y el área de concentración de éste, la cantidad y el enfoque de los refuerzos de borrones y las líneas cambiadas., ${ }^{5,12,14}$

Entre las interpretaciones generales que se han dado a los dibujos están:

- La fuerza del trazo demuestra mayor o menor agresividad.

- El tamaño de las orejas, en la figura humana, refleja su sensibilidad.

- La posición de la hoja deja ver el lugar en el tiempo; izquierda pasado, derecha futuro, arriba sueños y abajo realidad.

- El suelo indica seguridad al igual que los zapatos.

- Los brazos abiertos señalan necesidad de afecto.

- Se debe indagarz en el caso en que se omita papá o mamá o se pinten fuera de la familia.

- El pintarse entre papá y mamá sugiere que se siente dentro de la familia.

- Si se dibuja lejos o fuera significa rechazo., ${ }^{9,12-15}$

\section{Según el tipo de dibujo se podría argumentar:}

Dibujos geométricos: son fríos, impersonales; predomina el ángulo. Es muy frecuente en personas de carácter obsesivo. Generalmente, en personas que necesitan del orden, de lo metódico para sentirse confiados y "relajados".

Dibujos geométricos más sombreados: cuando los mismos dibujos son dibujados con sombras y arreglos decorativos: persona delicada que trata de suavizar asperezas

Dibujos en los que se aprecian cuadros blancos y negros: se trata de una naturaleza algo ambivalente, conducta con posibles contradicciones. Persona muy ordenada en sus cosas, con evidente orden tipo clasificatorio. Lo blanco es lo mental, la ansiedad, la inactividad; lo negro es la actividad, la acción.

Trazos rígidos con minuciosidad y repetición: dificultades para relajarse. Concentración angustiosa que termina en fatiga y agotamiento.

Puntos marcados con fuerza: carácter explosivo, con detalles de agresividad mal controlada y hasta asomos de violencia. Impulsividad.

Puntos marcados y adornados o decorados posteriormente: agresividad fuerte pero controlada. Equivalente de formación reactiva.

Rasgos marcados hasta llegar a agujerear el papel sobre el que se han hecho: excesiva violencia, frecuente cólera y carácter

124

Ustasalud Odontología 2006; 5:123 - 131

Reyes ES. y col. 
destemplado, con pérdida de control. Frecuente en niños muy activos o dinámicos que no soportan la idea de que algo no les salga como ellos esperan.

Lineas quebradas, zig-zag:la vida pasional y afectiva es fuerte, hay alternativas de excitación, control y calma. El sujeto acumula tensiones y luego las descarga intempestivamente.

Dibujar el sol, radiante: símbolo paterno y probables buenas relaciones con la figura del padre o imagen paterna. Vinculo idealizado o al menos positivo con la instancia paterna.

Dibujar un sol entre nubes: conflictos o problemas con la figura paterna.

Sol oscurecido: muerte real o «ha muerto para mí el padre o la figura paterna, rotura con el padre y lo que él representa.

Vehículos, barcos, coches, motos, bicicletas, aviones: deseos de cambio y de novedad.

Dibujos de macetas con flores y plantas decorativas: suavidad, dulzura, reposo. Deseos de agradar, personas que buscan conciliar y evitar confrontación con el medio.

Animales pequeños, gallinas, pájaros: se suele dar una mezcla de sensualidad y ansias de maternidad.

Dibujos de figuras humanas en general: son siempre, o casi siempre, autorretratos que expresan alguna preocupación por él mismo Vienen a ser siluetas del que dibuja.

Perfil mirando a la derecha: deseos de salir de si mismo, sociabilidad, extroversión. Preocupación por el propio fisico, por su salud.

Perfil mirando hacia la izquierda: introversión, retraimiento social.

Dibujo extraño, abstractos: busca la solución a sus problemas actuales en la huida, en el ensueño, fuera de la realidad. Evasividad, en general.

Dibujos esquemáticos, figuras palito: encubrimiento de la personalidad, frío y razonador. No pone el corazón en sus relaciones con los demás.

Dibujos de espadachines: crisis agresivas, sensuales y sexuales.

Dibujos de cabelleras: pelo lacio, corto, próximo a la cabeza: recato, modestia, encubrimiento de lo sexual. Cuidada, bien peinada, con algún adorno: coquetería, vanidad, deseos de agradar. Cabellos desordenados, exuberantes, en cascada: sensualidad. ${ }^{1216}$

El dibujo es importante pero no definitivo. Se debe hablar con el niño para que explique diferentes aspectos del dibujo y aclare las razones del mismo. La mayoría de dibujos se hacen para una persona específica y se expresan pensando en ella., ${ }^{5,6}$ En este caso, no aplica la frase "una imagen vale más que mil palabras" ya que es necesario que se logra obtener una mayor y veraz información cuando el niño explica lo que ha dibujado. ${ }^{6}$

Por lo tanto, se considera que el dibujo es una de las herramientas más versátiles de la que pueden disponer los profesionales de la salud. ${ }^{510,11,14} \mathrm{El}$ propósito de este trabajo fue analizar los rasgos generales de la personalidad y la percepción del paciente niño hacia el odontopediatra, en la consulta odontológica, a través de los dibujos realizados por los niños atendidos en la Clínica del Preescolar y del Escolar de la Especialización en Odontopediatría de la Universidad Santo Tomás.

\section{MATERIALES Y MÉTODOS}

\section{Hipótesis}

El dibujo realizado por niños de 6 a 9 años de edad, antes y después de la consulta odontológica, puede ser un medio positivo para el proceso de adaptación, manejo de la ansiedad, comportamiento y como medio de comunicación asertiva entre el odontopediatra y el paciente

\section{Grupo de estudio}

La muestra estuvo compuesta por 9 pacientes, 4 niñas y 5 niños, de estrato socioeconómico medio, que asistieron a la Clínica del Preescolar y del Escolar en la Universidad Santo Tomás de Floridablanca, durante los meses abril y mayo de 2005. La edad promedio de los pacientes fue 7.5 años. Los niños no presentaban limitaciones fisicas y mentales. Todos los pacientes tenían buenas experiencias odontológicas previas.

\section{Metodología}

A los niños les fueron repartidas hojas de papel y lápices de colores, en el momento de llegar a la clínica. Se les pidió que realizaran un dibujo libre y se solicitó a los padres que no interfirieran en la realización de éste. Posteriormente, el niño entregó el dibujo a la residente de odontopediatría quien realizó un procedimiento de operatoria simple, sin aplicación de anestesia local.

La técnica de manejo del comportamiento empleada fue diga, muestre y haga. ${ }^{1.3}$ Se determino el tipo de comportamiento de acuerdo con la Escala de Frankl. ${ }^{17} \mathrm{La} \mathrm{duración} \mathrm{del} \mathrm{procedimien-}$ to no excedió los 20 minutos. Una vez finalizado el procedimiento, se solicitó al niño que realizara un nuevo dibujo relacionado con la consulta odontológica.

Los dibujos fueron analizados por las cinco residentes de odontopediatría con asesoría sicológica y se registraron los resultados. 


\section{RESULTADOS}

\section{Dibujos, paciente número 1:}

- Edad de la paciente: 9 años.

- Género: Femenino.

- Tipo de paciente: Cooperador.

- Comportamiento en la consulta $(++)$

DIBUJO PREVIO A LA CONSULTA

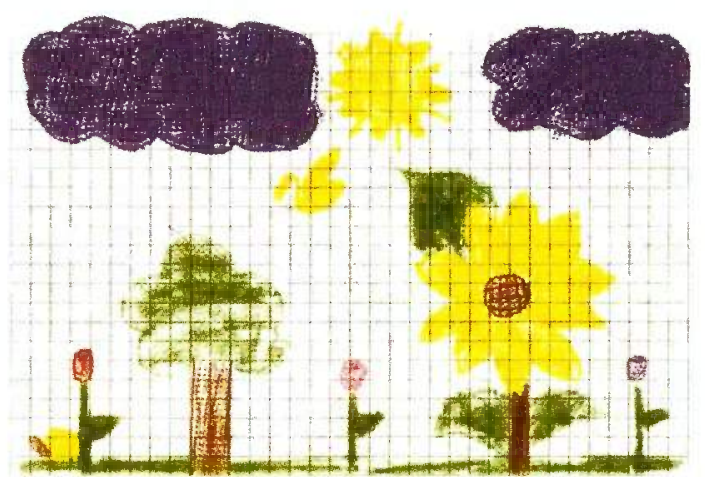

Las flores son cambios, es una niña que pronto iniciará su etapa prepuberal pero al mismo es tierna, amigable y sociable.

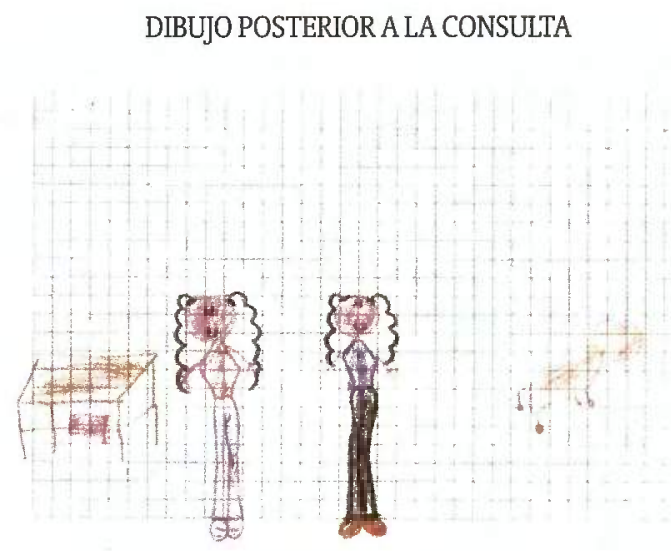

Silla distante, puede reflejar temor. Se identifica con la odontóloga, confía en ella pero no quiere estar cerca de la silla. Línea de igualdad con la odontóloga, buena relación con ella.

\section{Interpretación y análisis de los dibujos 1 y 2:}

Relación positiva, canaliza el temor específico a nivel del tratamiento. Proceso de socialización adecuado, indispensable para la edad de desarrollo en la que se encuentra. A pesar de ser una niña cooperadora parece reflejar malas experiencias odontológicas anteriores al dibujarse lejos de la silla, se debe tratar de disminuir la ansiedad para que responda mejor a la consulta.

\section{Dibujos, paciente número 2 :}

- Edad de la paciente: 9 años.

- Género: Femenino.
- Tipo de paciente: Cooperador.

Comportamiento en la consulta $(++)$

$$
\text { DIBUJO PREVIO A LA CONSULTA }
$$
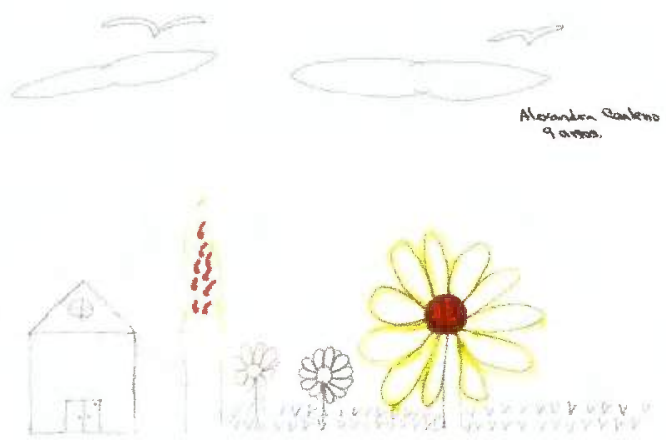

Las flores reflejan cambios. Las nubes reflejan sueños e ideales.

\section{DIBUJO POSTERIOR A LA CONSULTA}
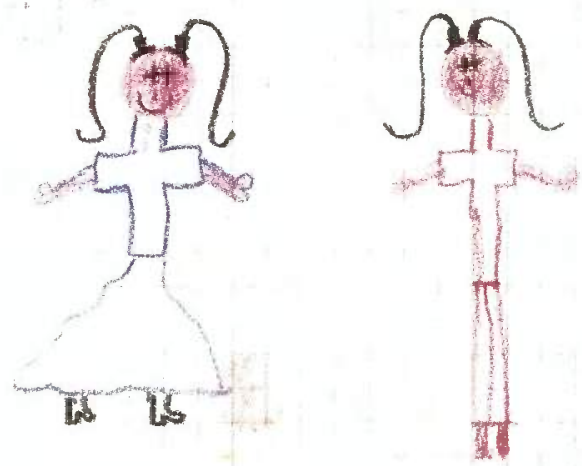

Hay una relación de empatía, la perfección en los trazos representa un nivel de exigencia, de hacer las cosas bien.

\section{Interpretación y análisis de los dibujos 1 y 2:}

Se refleja empatia, esta en el mismo nivel de la odontóloga, los brazos abiertos reflejan la disposición para establecer una relación asertiva, la necesidad de afecto; no parece temerosa. No dibujo la silla, puede indicar que para ella, tal vez, fue más importante la relación con la odontóloga que el tratamiento en sí.

3. Dibujos, paciente número 3 :

- Edad del paciente: 6 años.

- Género: Masculino.

- Tipo de paciente: Cooperador.

Comportamiento en la consulta $(++)$ 


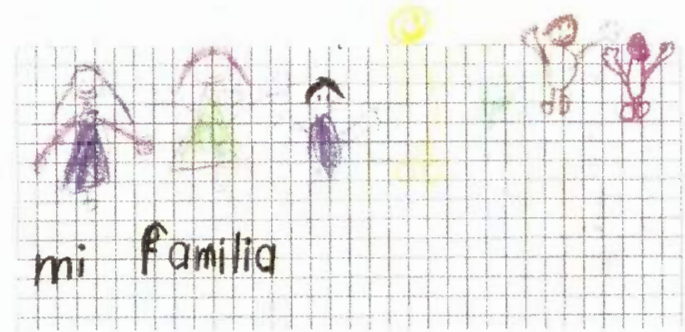

Idealización de la familia, vive situaciones que no quiere aceptar y tiende a idealizar reflejando lo que anhela.

DIBUJO POSTERIOR A LA CONSULTA

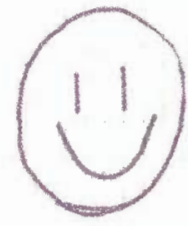

$$
\text { me Gusto bas }
$$$$
\text { sonrrisas de tadas }
$$

Se sintió bien en la consulta.

\section{Interpretación y análisis de los dibujos 1 y 2:}

Refleja ser observador, pues escribió en el dibujo, no sólo, las expresiones de su odontóloga sino de todas las demás. Le gustó la sonrisa que expresaban las odontólogas.

\section{Dibujos, paciente número 4:}

- Edad del paciente: 7 años.

- Género: Masculino.

- Tipo de paciente: Cooperador.

- Comportamiento en la consulta $(++)$

DIBUJO PREVIO A LA CONSULTA

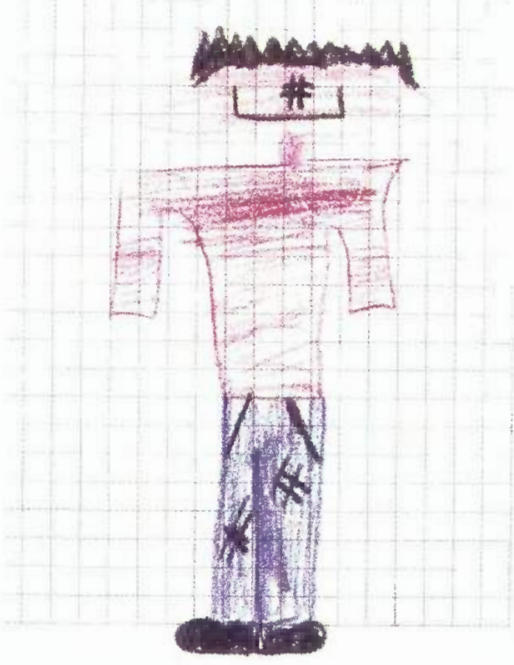

El color rojo simboliza la emotividad, las puntas agresividad, la ausencia de manos refleja algún tipo de agresión. Puede identificarse como él mismo quisiera ser.

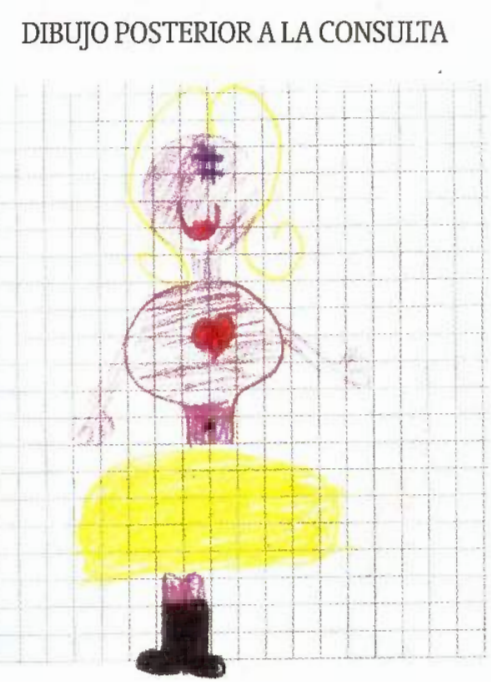

La ropa diferente simboliza que el niño quisiera ver diferentes colores. El corazón simboliza enamoramiento y las manos extendidas indican una necesidad de cariño.

\section{Interpretación y análisis de los dibujos 1 y 2:}

En ambos dibujos plasmo cosas similares, los dos personajes no tienen ojos ni nariz, al primero le faltan las manos. Estos pueden ser referentes emocionales.

\section{Dibujos, paciente número 5 :}

Edad de la paciente: $\quad 6$ años.

- Género: Femenino

- Tipo de paciente: Cooperador.

Comportamiento en la consulta $(++)$

\section{DIBUJO PREVIO A LA CONSULTA}


La niña pinta una casa distante y bajo la lluvia. Se destaca un osito al que le llegan los rayos del sol, puede significar una relación distante con sus padres y es a su osito a quien le manifiesta sus sentimientos. Se pinta en el extremo inferior.

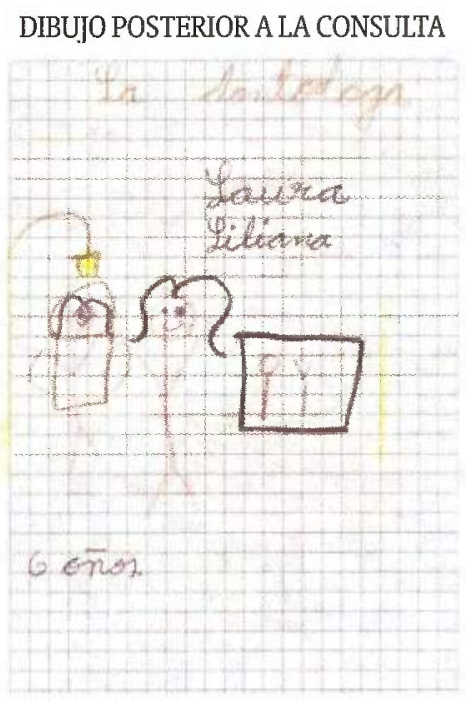

Tiene muy buena relación con la odontóloga, le gusta ir a la consulta y se siente bien allí. Se dibuja feliz sentada en la unidad odontológica y ninguno de los implementos utilizados le causa temor.

\section{Interpretación y análisis de los dibujos 1 y 2:}

Dibujarse en el extremo inferior de la hoja puede manifestar que se siente poco importante en su casa o dentro de su familia. El segundo dibujo permite interpretar que es una niña bastante colaboradora en la consulta y a la que se puede tratar fácilmente.

\section{Dibujos, paciente número 6:}

- Edad de la paciente: 8 años.

- Género:

- Tipo de paciente: Cooperador.

- Comportamiento en la consulta $(++)$

DIBUJO PREVIO A LA CONSULTA

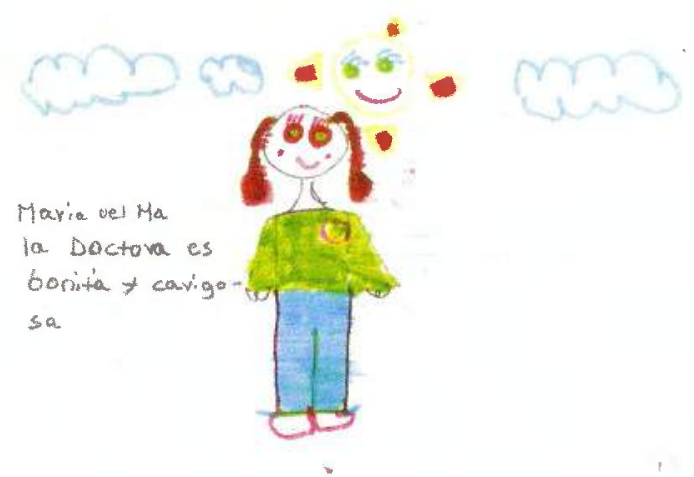

Se pinta cercana al sol con mucho colorido. Refleja sus sueños.

$$
\text { DIBUJO POSTERIOR A LA CONSULTA }
$$

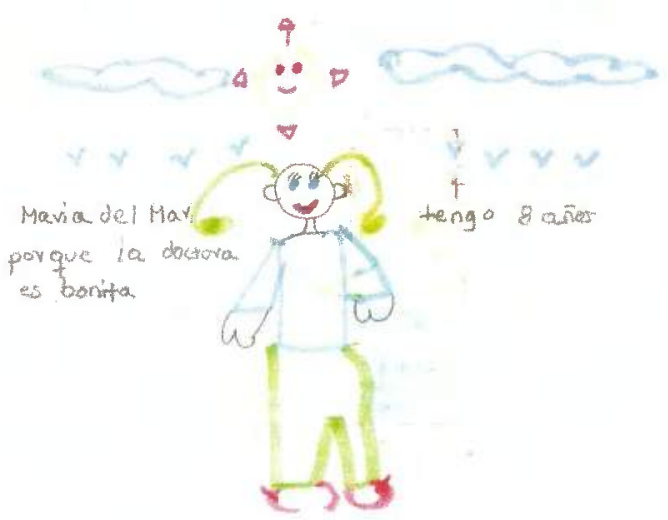

El dibujo es similar al anterior.

\section{Interpretación y análisis de los dibujos 1 y 2:}

Tiene sueños, relación cercana con su padre. Trazos con mucho detalle y dibujos centrados, tiene manos, brazos, muchos colores, no demuestra carencia de afecto y con muchos rasgos faciales.

\section{Dibujos, paciente número 7:}

- Edad del paciente: 7 años.

- Género: Masculino

- Tipo de paciente: Cooperador

- Comportamiento en la consulta $(++)$

\section{DIBUJO POSTERIOR A LA CONSULTA}
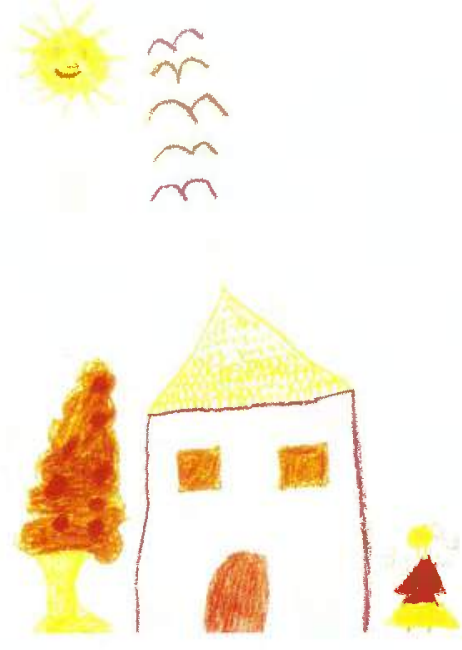

No define muy bien las personas, pinta la casa como figura principal. Muestra una figura de niña en el extremo derecho inferior. Sin manos y sin expresiones faciales. En el extremo superior izquierdo se observa una figura del sol bien elaborada con rasgos faciales definidos. 


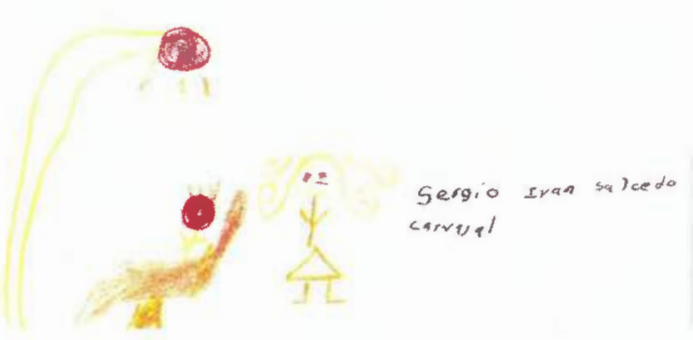

Sigue sin definir los rasgos aunque parece dibuja más a la odontopediatra. Dibuja la luz y sus "rayos".

\section{Interpretación y análisis de los dibujos 1 y 2:}

Los pájaros forman una barrera que lo alejan de la figura paterna. La odontóloga esta situada detrás de él, rasgos faciales fuertes, pero con brazos abiertos. El se dibujo sin rasgos faciales y con brazos abiertos. Relación distante pero él es receptivo a cualquier cambio en el proceso, lo cual, es importante porque a pesar de no tener una relación muy estrecha con la odontóloga, se puede lograr una buena consulta.

\section{Dibujos, paciente número 8:}

- Género: Masculino

- Tipo de paciente: Cooperador.

- Comportamiento en la consulta $(++)$

DIBUJO PREVIO A LA CONSULTA

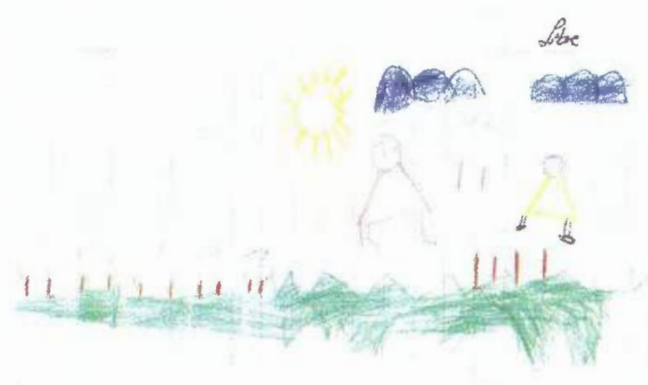

Se dibuja en la parte superior derecha, muestra trazos puntiagudos que pueden indicar un cierto grado de agresividad. Las figuras humanas no están, claramente, diseñadas; carecen de brazos, boca, manos, ojos, nariz y se encuentran flotando. Los dibujos corresponden a él y a su hermano jugando en el parque.

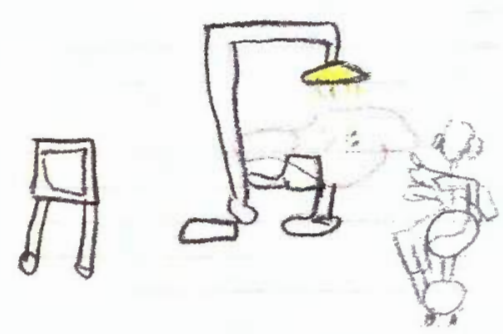

$9=$

Sólo se aprecian sus rasgos faciales, unos ojos muy abiertos, que pueden significar gran atención y alta percepción de todo cuanto ocurre entorno a la cita odontológica. Se pintó en la unidad odontológica en el centro de ella pudiendo indicar que él es importante en la consulta, aunque pueda sentir temor hacía algunos procedimientos.

\section{Interpretación y análisis de los dibujos 1 y 2:}

Se hace importante tener en cuenta dicho temor para una próxima sesión dando seguridad al paciente, reforzando el comportamiento positivo y elogiando al niño por su cooperación.

\section{Dibujos, paciente número 9:}

- Edad del paciente: 9 años.

- Género: Masculino

- Tipo de paciente: Cooperador.

- Comportamiento en la consulta $(++)$

DIBUJO PREVIO A LA CONSULTA

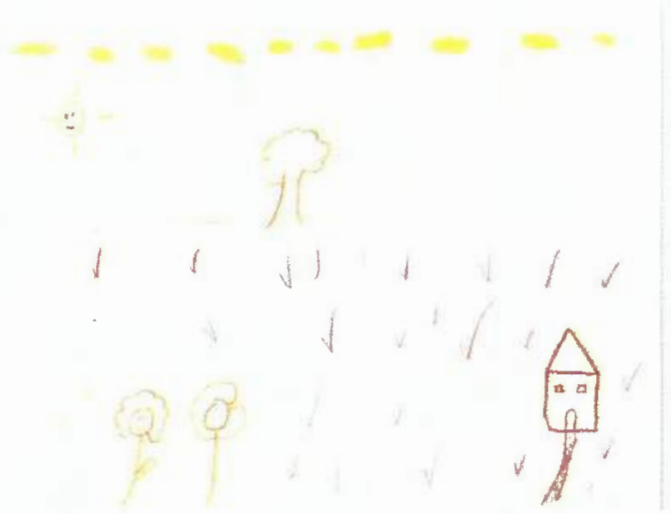

Presenta un dibujo libre del campo, donde hay flores, árboles, nubes, personas pescando, parece tener amor por la naturaleza y relación cercana con el campo. 
DIBUJO POSTERIOR A LA CONSULTA

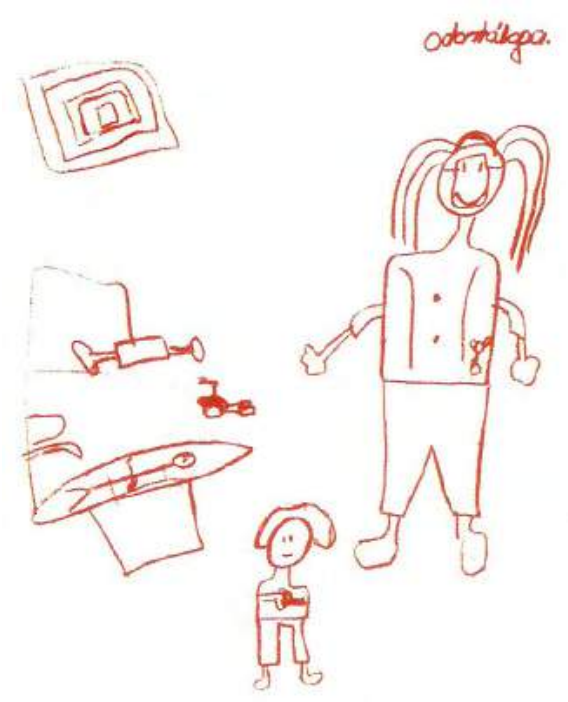

Es detallista, pinta todo lo observado en el espacio donde se encontraba. Al parecer su mamá no es tan importante durante la consulta.

\section{Interpretación y análisis de los dibujos 1 y 2:}

Aparenta ser un niño soñador, sus dibujos son pequeños a excepción de las flores que denotan cambios. A nivel odontológico, el niño se dibuja pequeño en la silla, puede ser por temor. El dibujo de la odontóloga es muy grande lo que puede reflejar un "dominio" por parte de ella en la consulta.

\section{DISCUSIÓN}

En todos los dibujos se apreció una buena percepción frente a la consulta odontológica y frente a las estudiantes de la Especialización en Odontopediatría. Esto puede ser, en parte, debido a que los niños mostraban un comportamiento positivo, los procedimientos realizados fueron sencillos y la técnica de manejo del comportamiento utilizada fue didáctica.

El dibujo realizado, antes de la consulta odontológica, produce relajación y agrado en el niño. Éste ayuda a atravesar la barrera de la incertidumbre de lo que sucederá en la consulta. ${ }^{5}$ Sin embargo, es importante recalcar que no es conveniente considerar el dibujo como una herramienta por sí misma, es decir, se requiere un dialogo con el niño para establecer las razones que lo llevaron a dibujar lo que pinto. ${ }^{6}$

Se ha sugerido que los niños ansiosos dibujan objetos más pequeños que los niños que no experimentan esta sensación, tal vez debido a un posible mecanismo de defensa. ${ }^{10,11}$ Burkitt y colaboradores evaluaron 258 niños y observaron que los niños dibujaron las figuras más grandes cuando eran percibidas de manera positiva, y más pequeñas si les eran negativas. ${ }^{7}$ Aunque este concepto sobre un posible mecanismo de defensa es discutido por Jolley y Vulic-Prtoric. ${ }^{18}$ Estos autores no encontraron asociaciones significativas entre el tamaño del dibujo y su ubi- cación espacial con los efectos emocionales que el niño desarrolla ante un evento que le cause ansiedad; este estudio fue realizado con 60 niños croatas, después de la guerra ${ }^{18}$

En algunos de los dibujos realizados por los niños que participaron en este estudio, se observa que la silla odontológica es dibujada lejos (Paciente 1, 9 años) o muy pequeña (Paciente 9, 9 años). Se podría sugerir que estos pacientes no ven como placentera la consulta odontológica. En otros dibujos, no fue tomada en cuenta, tal vez, porque fue más relevante el profesional que el objeto en sí.

Con respecto al el uso de los colores, Burkitt y colaboradores observaron que los colores oscuros (negro, café) son utilizados para dibujar las situaciones que no son agradables. ${ }^{8}$ Aunque aclaran que es necesario indagar si estos mismos colores son usados por niños de todas las razas. Milne y Greenway sugieren que las niñas usan más colores que los niños en sus dibujos y que éstos son integrados en una edad más temprana. ${ }^{9}$ En este trabajo, los dibujos realizados por las niñas fueron más coloridos que los dibujos realizados por los niños.

En relación con las edades de los niños participantes en el trabajo, todos presentaban las habilidades para dibujar una figura humana. Sin embargo, en algunos casos se omitieron los ojos, la nariz, la boca, las manos. Estas omisiones pueden estar de acuerdo con el desarrollo esperado de acuerdo para su edad (Tabla 1) pero en otras no. ${ }^{14}$ Puede ser que el niño y/o sus acompañantes hayan tomado a la ligera la realización del dibujo o que, realmente, sean indicadores emocionales. Por ejemplo, en el dibujo del paciente 4 no están las manos, ni la nariz lo que podría indicar timidez, agresividad, culpa.

Adicionalmente, el dibujo posterior a la consulta se convierte en una manera para evaluar el quehacer profesional y su influencia sobre el niño. ${ }^{5}$ Esta actividad es de vital importancia para realizar una retroalimentación de la propia conducta del profesional de la salud ante el comportamiento del paciente pediátrico.

Dentro de las limitantes del trabajo se pueden considerar, la carencia de diálogo con el niño para indagar sobre el significado de cada dibujo, el por qué lo realizó de esa manera, qué quiso transmitir a través de él. Adicionalmente, no se tuvo en cuenta que todas las hojas en las que se realizaron los dibujos fueran iguales. Sin embargo, con este trabajo se abre una puerta para que los odontopediatras conozcan y estudien sobre las posibilidades que implica un dibujo infantil y sobre cómo en otras profesiones ya se esta indagando, ampliamente.

El odontopediatra podría incursionar en una nueva técnica de manejo del comportamiento del niño basada en un acercamiento inicial a través de un método sencillo, económico, divertido y que le puede proporcionar información para lograr una mejor relación con el paciente aún antes de que éste se siente en el sillón odontológico. No sólo se lograría este objetivo sino que, también, a través del dibujo se estimula el desarrollo y la creatividad del paciente pediátrico. 
Tabla 1. Zonas esperadas que sean dibujadas en el $85 \%$ de los niños de acuerdo con el desarrollo para cada grupo de edad. ${ }^{14}$

\begin{tabular}{ccccc}
\hline 5 años & 6 años & 7 años & 8 años & 9 años \\
\hline Cabeza & Todo lo anterior & Todo lo anterior & Todo lo anterior & Todo lo anterior \\
Ojos & BrazosBrazos & BrazosBrazos & \\
Nariz & Pies & Pies Pies & \\
Boca & Brazos en 2D & Brazos en 2D & Brazos en 2D \\
Cuerpo & & Piernas en 2D & Piernas en 2D \\
Piernas & & & \\
\hline 2D = Segunda dimensión. & & & \\
\hline
\end{tabular}

\section{CONCLUSIONES}

1. El dibujo, en la consulta odontológica, es una buena herramienta para mejorar el proceso de adaptación del menor, el manejo de la ansiedad y el manejo del comportamiento.

2. El dibujo inicial ayuda a que el niño libere parte de la ansiedad que genera la consulta odontológica y facilita su receptividad hacia las técnicas de manejo lo que mejora su comportamiento durante la consulta.

3. El dibujo posterior a la consulta es una retroalimentación y auto-evaluación del odontólogo. Sirve para identificar fortalezas y debilidades en el manejo de comportamiento y en la relación odontólogo-paciente. Además, es muy útil para identificar los elementos de la consulta que le generan temor 0 ansiedad y los que los que le agradaron.

4. Ningún dibujo por si sólo es evidencia concluyente de la personalidad del niño. Es muy importante generar un espacio de dialogo donde el niño pueda explicar lo que representa su dibujo.

\section{AGRADECIMIENTOS}

A la Dra. Bertha Latorre Gómez por la orientación psicológica en la realización de este trabajo. A la Dra. Martha Juliana Rodríguez Gómez por la revisión y la corrección del manuscrito.

\section{BIBLIOGRAFÍA}

1. Cárdenas JM. Pautas para el manejo no farmacológico del comportamiento en el paciente pediátrico. Revisto de la Academia Colombiana de Odontología Pediátrica 1997,1: 30 - 34.

2. Torres EA Rodríguez MJ Cañón OL. Manejo del comportamiento del paciente niño en la consulta odontológica. Ustasalud 2002; 1: 49 - 56.

3. Cárdenas JM. Manejo del comportamiento del paciente pediátrico. En: Cárdenas D. Odontología pediátrica. 3ra ed. Medellín: Corporación para la Investigación Biológica; 2003. p. 22 - 34.

4. Muñoz N, Álvarez LM, Cárdenas JM. Eficacia de la técnica de distracción contingente con material auditivo en el comportamiento de pacientes entre 4 y 6 años de edad. CES Odontología 1998; 11: 13 - 19.
5. Ryan-Wenger NA. Children's drawings: An invaluable source of information for nurses. Editorial. J Pediatr Health Care 1998; 12: 109 - 110.

6. Driessnack M. Children's drawings as facilitators of communication: A meta-analysis. J Pediatr Nurs 2005; 20: 415 - 423.

7. Burkitt $E$, Barrett $M$, Davis A. The effect of affective characterizations on the size of children's drawings. Br J Develop Psychol 2003; 21: 565 - 584 .

8. Burkitt E, Barret M, Davis A. Children's colour choices for completing drawings of affectively characterised topics. J Child Psychol Psychiatry 2003; 44: 445 - 455.

9. Milne LC, Greenway P. Color in children's drawings: The influence of age and gender. The Arts in Psychoterapy 1999; 26: $261-263$.

10. Balwin DC Jr. An investigation of psychological and behavioural responses to dental extraction in children. J Dent Res 1966; 45: 1637-1651.

11. Puura A, Puura K, Rorarius M, Annila P, Viitanen H, Baer G. Children's drawings as a measure of anxiety leve: a clinical pilot study. Pediatr Anaesthesia 2005; 15: 190 - 193.

12. Lowenfeld V, Brittain LW. Desarrollo de la capacidad creadora. Kapelusz; Buenos Aires: 1980.

13. Conde Jerez A. Las vanguardias del siglo XX en relación el dibujo infantil. Alonso Cano Revista Andaluza de Arte 2004; 1: 1 - 11.

14. Tielsch AH, Jackson P. Listen to them draw: Screening children in primary care through the use of human figure drawings. Pediatr Nurs 2005; 31: $320-327$.

15. Dunn J, O'Connor T, Levy I. Out of the picture: A study of family drawings by children from step-, single-parent, and non-step families. J Clin Child Adolesc Psychol 2002; 31: 505- 512.

16. Análisis de los dibujos inconciente y garabatos espontáneos. URL disponible en: http://www.angeifire.com/ak/psicologia/2.html

17. Frankl S, Shiere F, Rogels H. Should the parent remain with the child in the dental operatory. J Dent Child 1962; 29: 150 - 161.

18. Jolley R, Vulic-Prtoric A. Croatian children's experience of war is not reflected in the size and placement of emotive topics in their drawings. Br J Clin Psychol 2001; 40: 107 - 110. 